\title{
A Circuit Model for CMOS Hall Cells Performance Evaluation including Temperature Effects
}

\author{
Maria-Alexandra Paun, ${ }^{1,2}$ Jean-Michel Sallese, ${ }^{1}$ and Maher Kayal ${ }^{1}$ \\ ${ }^{1}$ STI-IEL-Electronics Laboratory, Ecole Polytechnique Fédérale de Lausanne (EPFL), 1015 Lausanne, Switzerland \\ ${ }^{2}$ Department of Engineering, University of Cambridge, Cambridge CB2 1PZ, UK \\ Correspondence should be addressed to Maria-Alexandra Paun; map65@cam.ac.uk
}

Received 28 May 2013; Revised 6 August 2013; Accepted 8 August 2013

Academic Editor: Jan Alexander Jung

Copyright (C) 2013 Maria-Alexandra Paun et al. This is an open access article distributed under the Creative Commons Attribution License, which permits unrestricted use, distribution, and reproduction in any medium, provided the original work is properly cited.

In order to provide the information on their Hall voltage, sensitivity, and drift with temperature, a new simpler lumped circuit model for the evaluation of various Hall cells has been developed. In this sense, the finite element model proposed by the authors in this paper contains both geometrical parameters (dimensions of the cells) and physical parameters such as the mobility, conductivity, Hall factor, carrier concentration, and the temperature influence on them. Therefore, a scalable finite element model in Cadence, for behavior simulation in circuit environment of CMOS Hall effect devices, with different shapes and technologies assessing their performance, has been elaborated.

\section{Introduction}

Hall effect sensors are largely used in the actual industrial context as magnetic sensors aimed primarily at sensing the current in a large variety of applications, and also for proximity switching, positioning, or speed detection [1]. The sensitivity, offset, and their temperature drift are important parameters that dictate the performance of Hall effect sensors.

There is a strong connection between the geometry of the Hall device and the performance [2]. For Hall effect sensors performance analysis, three-dimensional physical models were constructed and simulation results were reported by the authors [3]

To achieve high sensitivity, small offset, and drift, various Hall effect sensors configurations were integrated in a CMOS technology. Extensive measurements on the new proposed cells [4] proved that with specific shapes (XL) we managed to reach room temperature offset less than $30 \mu \mathrm{T}$ and $0.039 \mu \mathrm{T} /{ }^{\circ} \mathrm{C}$ drift, which is 3-4 times lower than the state of the art.

The motivation of the current work is to provide a circuit model able to predict the temperature effects on the Hall effect sensors and their influence on the performance. Under the assumed research objective, a different elementary cell, with a slightly modified design, including the temperature effects, is proposed and implemented in Cadence.

Section 2 focuses on presenting the Hall effect sensors integration, providing the basic equations related to these magnetic sensors. Within this section, a Hall sensors analysis by depicting the measurements results is also performed and the shape providing the best results is identified. The XL cell designed and integrated by the authors proved to have the lowest offset and offset temperature drift.

Section 3 aims to introduce the new simpler elementary cell to be used in the lumped circuit model. In this case, there are two independent magnetic and electric paths. The number of elementary cells in the FEM model can be varied according to the accuracy level which needs to be achieved. However, for rough estimation of the Hall voltage and sensitivity, a single elementary cell can also be used. Details on the Hall cells polarization are also given at this point.

In Section 4, the FEM simulations results are presented and interpreted for three different Hall cells, with information on the Hall voltage and absolute sensitivity. In this section, 
TABLE 1: Specific parameters evaluation for some of the integrated Hall cells.

\begin{tabular}{|c|c|c|c|}
\hline Integrated geometry & $\mathrm{XL}$ & Optimum & Borderless \\
\hline Shape & שם & $\begin{array}{ll}\square & \square \\
\square & \square\end{array}$ & $\begin{array}{ll}\square & \square \\
\square & \square \\
\end{array}$ \\
\hline $\begin{array}{l}\text { Measured } R(\mathrm{k} \Omega) \\
@ T=300 \mathrm{~K}, B=0 \mathrm{~T}\end{array}$ & 2.2 & 1.8 & 1.3 \\
\hline $\begin{array}{l}\text { Measured } S_{I}(\mathrm{~V} / \mathrm{AT}) \\
@ I_{\text {bias }}=1 \mathrm{~mA}\end{array}$ & 80.6 & 62.4 & 31.1 \\
\hline $\begin{array}{l}\text { Measured offset drift } \\
\left(\mu \mathrm{T} /{ }^{\circ} \mathrm{C}\right)\end{array}$ & 0.039 & 0.328 & 0.526 \\
\hline \multirow{2}{*}{$L, W(\mu \mathrm{m})$ of the $n$-well } & $L=43.2$ & $L=50$ & $L=50$ \\
\hline & $W=19$ & $W=50$ & $W=50$ \\
\hline Contacts length $(\mu \mathrm{m})$ & 18.3 & 4.7 & 2.3 \\
\hline
\end{tabular}

the emphasis is put on a particular Hall sensor in the shape of the XL cell with prediction of its current-related sensitivity temperature drift. Finally, Section 5 draws the conclusions.

\section{Hall Effect Sensors Integration}

2.1. Equations Related to Hall Effect Sensors. The Hall voltage is defined as follows [5] for voltage polarization:

$$
V_{H}=G \mu_{H} \frac{W}{L} V_{\text {bias }} B
$$

or equivalently for current polarization as

$$
V_{H}=G \frac{r_{H}}{n q t} I_{\text {bias }} B
$$

where $G$ is the geometrical correction factor, $\mu_{H}$ is the Hall mobility, $W$ and $L$ stand for the width and length of the device respectively, $V_{\text {bias }}$ is the voltage bias, $r_{H}$ is the Hall scattering factor, $n$ is the carrier concentration, $t$ is the thickness of the $n$-well implantation, and $B$ is the magnetic field on a direction perpendicular to the semiconductor probe.

The current-related sensitivity $S_{I}$ of a Hall effect sensor has the following analytical expression:

$$
S_{I}=\frac{V_{H}}{B I_{\text {bias }}}=G \frac{r_{H}}{n q t} .
$$

2.2. Hall Effect Sensors Analysis and Measurements Results. Different Hall effect sensors have been integrated in a $0.35 \mu \mathrm{m}$ CMOS technology and tested for their sensitivity, offset, and offset temperature drift. To this purpose, an automated measurement setup presented by the authors in [4] to test the integrated Hall effect sensors was used.

The following experimental results presented in Table 1, for three of these integrated Hall cells, were obtained. The design parameters (the length, width, and the contacts dimension) are indicated for every structure. For each device, the input resistance, as well as the current-related sensitivity and offset temperature drift measurements, is included in the

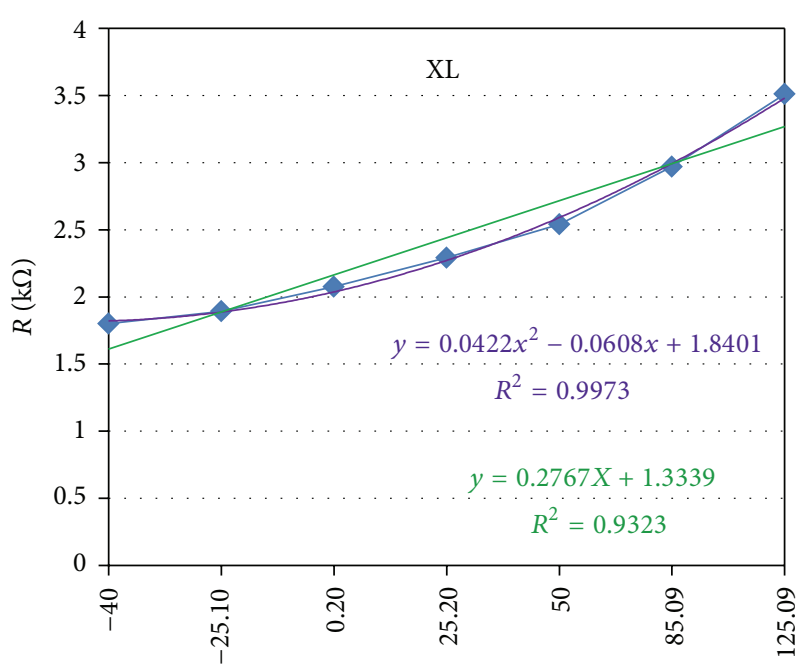

$T\left({ }^{\circ} \mathrm{C}\right)$

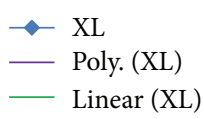

FIGURE 1: The measured input resistance versus temperature for XL cell.

table. We can observe that the XL cell presented the lowest offset drift.

Measurements for the resistance dependence with temperature of two integrated Hall effect sensors (XL and borderless cells) are depicted in Figures 1 and 2. The corresponding sensors were placed in an oven and the temperature was cycled from -40 to $125^{\circ} \mathrm{C}$. The curves are presented with both linear and second order fits.

In the subsequent graphs, Figures 1 and 2, the measured electrical input resistance $R$, computed as the regular ratio of voltage to current, are presented for two integrated Hall cells.

\section{The Hall Effect Sensors Lumped Circuit Model}

3.1. Elementary Cell Description. In order to be able to correctly investigate the Hall cells behavior and predict their performance, an FEM lumped circuit model was developed.

As it is known, a lumped circuit model, also named lumped element model, simplifies the behaviour description of spatially distributed physical systems. In fact, this model is used to recreate the topology of a specific physical system with the aid of discrete entities that would approximate its conduct. The advantages of the finite element model (FEM) consist in the possibility to test many different shapes with the aid of a single elementary cell and the fact that the desired accuracy can be tuned by the choice of the number of elementary cells. In addition, different integration processes can be simulated. The parameters used by the model are grouped in geometrical parameters (chosen by the designer) and technological parameters (specific to the fabrication 


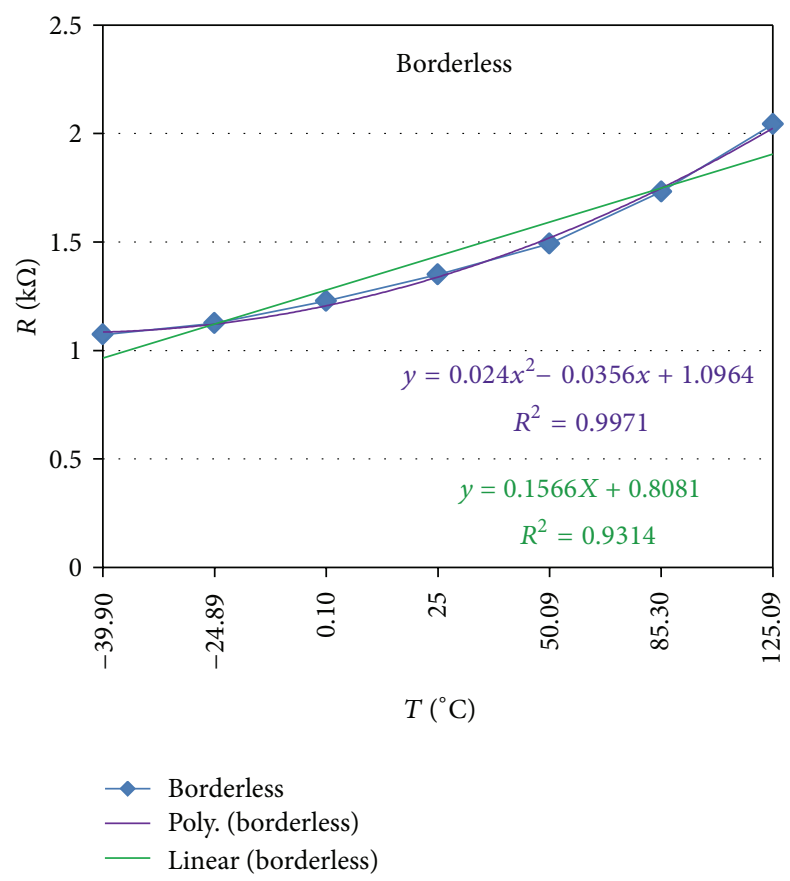

FIGURE 2: The measured input resistance versus temperature for borderless cell.

process). The latter can be either obtained by parameters extraction or by theoretical prediction where possible.

To model the Hall effect sensors, an FEM lumped circuit model containing a new elementary cell was developed. With respect to the previous papers introducing FEM lumped circuit models for Hall effect sensors [6], the new elementary cell proposed has a slightly modified design. In our case, we have two independent magnetic and electric paths.

The elementary cell in Figure 3 is a collection of currentcontrolled current sources $\left(F_{1}\right.$ and $\left.F_{2}\right)$ and parameterized resistances $\left(R_{X}\right.$ and $\left.R_{Y}\right)$. In order to sense the current flowing through each branch, DC sources with null voltage ( $V_{1}$ and $V_{2}$ ) were introduced. This elementary cell is modeled as a port with eight input/output pins, as in Figure 4.

To build the Hall cell, interconnections of several elementary cells will be used in order to recreate the layout of the structure. Therefore, the representation in Figure 5 is useful to see how the actual elementary cells are linked.

The resistances on each branch are given by the following equations, where $\sigma$ is the conductivity of the material:

$$
R_{X}=\frac{L}{t W \sigma}, \quad R_{Y}=\frac{W}{t L \sigma},
$$

while the currents $i_{X}$ and $i_{Y}$ are defined as follows:

$$
i_{X}=\frac{W}{L} \mu_{H} B i_{Y}, \quad i_{Y}=\frac{L}{W} \mu_{H} B i_{X} .
$$

By consequence, from the equation above, we can observe that each current flowing through a branch can be defined by the current through the opposite (orthogonal) branch multiplied by certain gains, $K_{Y X}$ and $K_{X Y}$, respectively, as follows:

$$
i_{X}=K_{X Y} i_{Y}, \quad i_{Y}=K_{Y X} i_{X},
$$

where the specific gains are introduced in the following way:

$$
K_{X Y}=\frac{W}{L} \mu_{H} B, \quad K_{Y X}=\frac{L}{W} \mu_{H} B .
$$

3.2. FEM Representation and Polarization of Hall Cells Using Interconnections of the Elementary Cell. The elementary cell in Figure 3 will be a part of the FEM lumped circuit model. In this way, the geometry of the Hall cell is reconstructed with a number of elementary cells. The interconnections and the current flow in the entire Hall cell will produce a certain geometrical correction factor specific to each structure.

The particular circuit model for the XL Hall cell is presented in Figure 5. A number of 64 elementary cells was used, but for visual purpose, only 12 are shown. On the borders, different cells could be added in the future to model possible asymmetries of the cell.

In the case of the Hall cell, the Hall voltage $V_{H}$ will be deducted from the current flowing through the currentcontrolled current sources multiplied by the corresponding parameterized resistance on that branch as

$$
V_{H}=R_{X} i_{X}=R_{Y} i_{Y} .
$$

High impedances $\left(Z_{\text {high }}=1 \mathrm{G} \Omega\right)$ were also placed at the end of the electric path which is not biased in order to force the current to flow only in the desired direction.

In order to polarize the Hall cell, we use current bias on the electric path, from left to right. Tensions will be created on the two independent magnetic paths, in the form of a $V_{H_{+}}$and $V_{H_{-}}$, respectively. The Hall voltage is therefore the difference of these two potentials

$$
V_{H}=V_{H_{+}}-V_{H-} .
$$

This polarization scheme, as shown in Figure 5, is used for the simulation of the XL sensor, reconstructed by FEM.

3.3. Three-Dimensional Physical Simulations. To assess the performance of Hall effect sensors, three-dimensional physical simulations were also performed by the authors in recent papers [7-9].

The selection of either physical or circuit model is dictated by the objectives of the user. For example, when one needs to integrate in circuit environment the Hall cell, the lumped circuit model should be used. For detailed analysis of the physical behaviour, the three-dimensional simulations should be performed. The advantage of the latter is that they offer more faithful reproduction of the internal physical processes but require more time, whilst the circuit model's accuracy is given by the choice of the corresponding parameters and their physical association. Between the two models, the circuit model is definitely faster.

In Figure 6, a three-dimensional model of XL Hall cell developed by the authors [3] is also depicted. The dimensions, 


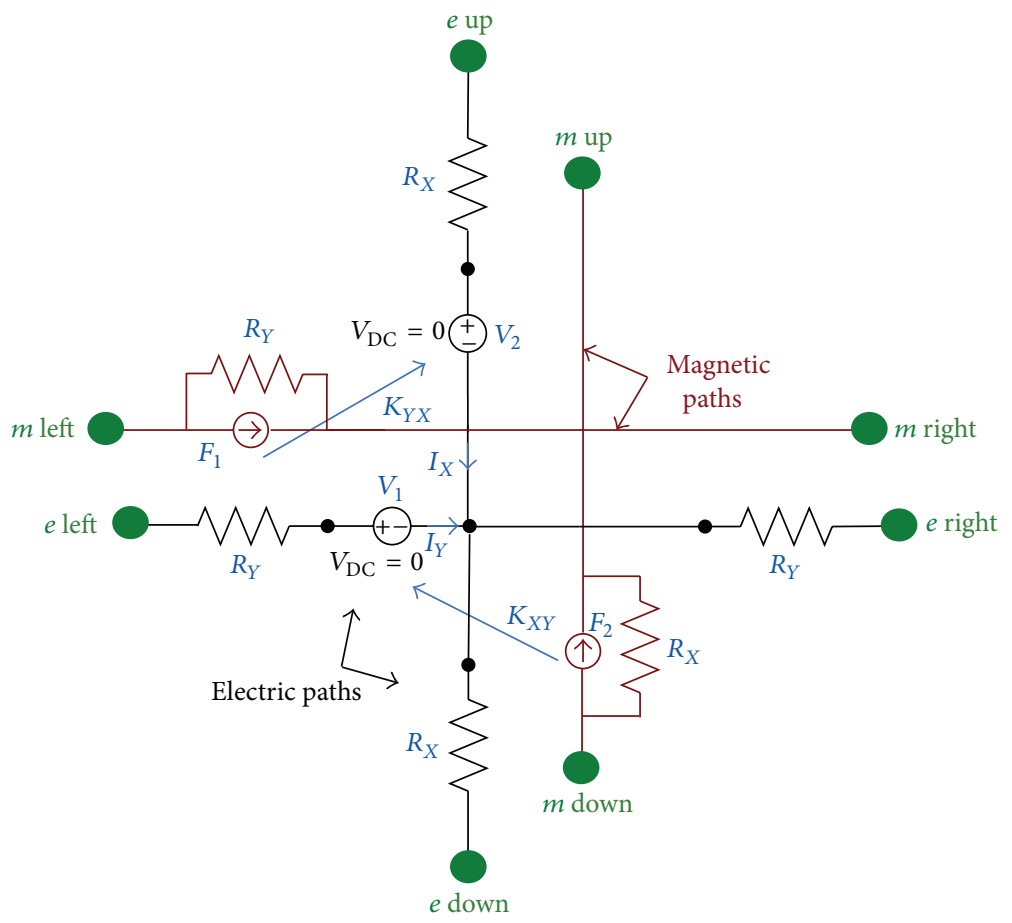

FIGURE 3: The elementary cell (e) model with its eight input/output pins highlighted.

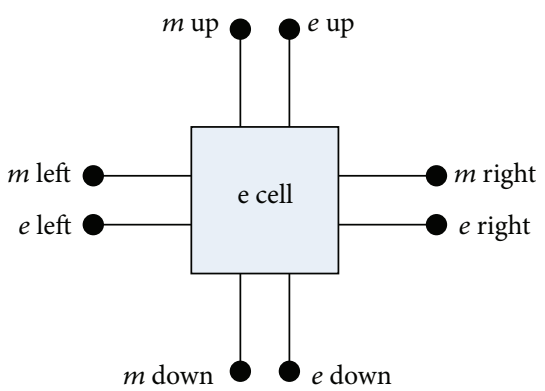

FIGURE 4: The elementary cell representation as a port with eight input/output pins.

position of contacts, and the electrostatic potential lines (for $1 \mathrm{~V}$ bias on contact $a$ ) are all shown on the simulated structure. Depending on the type of simulation that needs to be performed, either physical or circuit models should be used. There is coherence between the results from both physical and circuit simulations and the experimental results.

3.4. Temperature Effects on the Hall Cells Behavior. The Hall cells temperature behavior is of importance in their performance assessment. The temperature drift of the currentrelated sensitivity is of particular interest.

To be able to model the temperature dependence of the current-related sensitivity, one would need to take into account the parameters that vary with temperature from (3). As a first approximation, we could consider $t$ as constant, but $n$ and $r_{H}$ are both temperature dependent. A detailed analysis of the dependence of $n$ with temperature was performed including freeze-out effect (see Figure 7). We were mainly interested to model sensors such as the XL cell which displayed the best behavior and be able to predict its sensitivity and corresponding temperature drift.

The temperature dependence of the carrier concentration $n$ is described by the following relationship which includes the freeze-out effect:

$$
n= \begin{cases}n_{i}, & \text { if } n_{i}>\min \left(N_{d}-N_{a}, n_{f r}\right) \\ \min \left(N_{d}-N_{a}, n_{f r}\right), & \text { otherwise }\end{cases}
$$

where $n_{i}$ is the intrinsic carrier concentration, $N_{d}$ is the donor density, $N_{a}$ is the acceptor density, and $n_{f r}$ is the freeze-out concentration.

The temperature dependence of $n_{f r}$ follows the relation

$$
\begin{aligned}
n_{f r}(T)= & \left(\left(\frac{\left(N_{C} / 2\right) \mathrm{e}^{-E_{d} / k T}+N_{a}}{2}\right)^{2}\right. \\
& \left.+\left(N_{d}-N_{a}\right)\left(N_{C} / 2\right) \mathrm{e}^{-E_{g} / 2 k T}\right)^{1 / 2} \\
& -\frac{\left(N_{C} / 2\right) \mathrm{e}^{-E_{d} / k T}+N_{a}}{2}
\end{aligned}
$$

with $N_{C}$ being the effective density of states in the conduction band, $E_{d}$ the donor energy of the donor impurity, $E_{g}$ the bandgap of the semiconductor, $k$ the Boltzmann constant, 


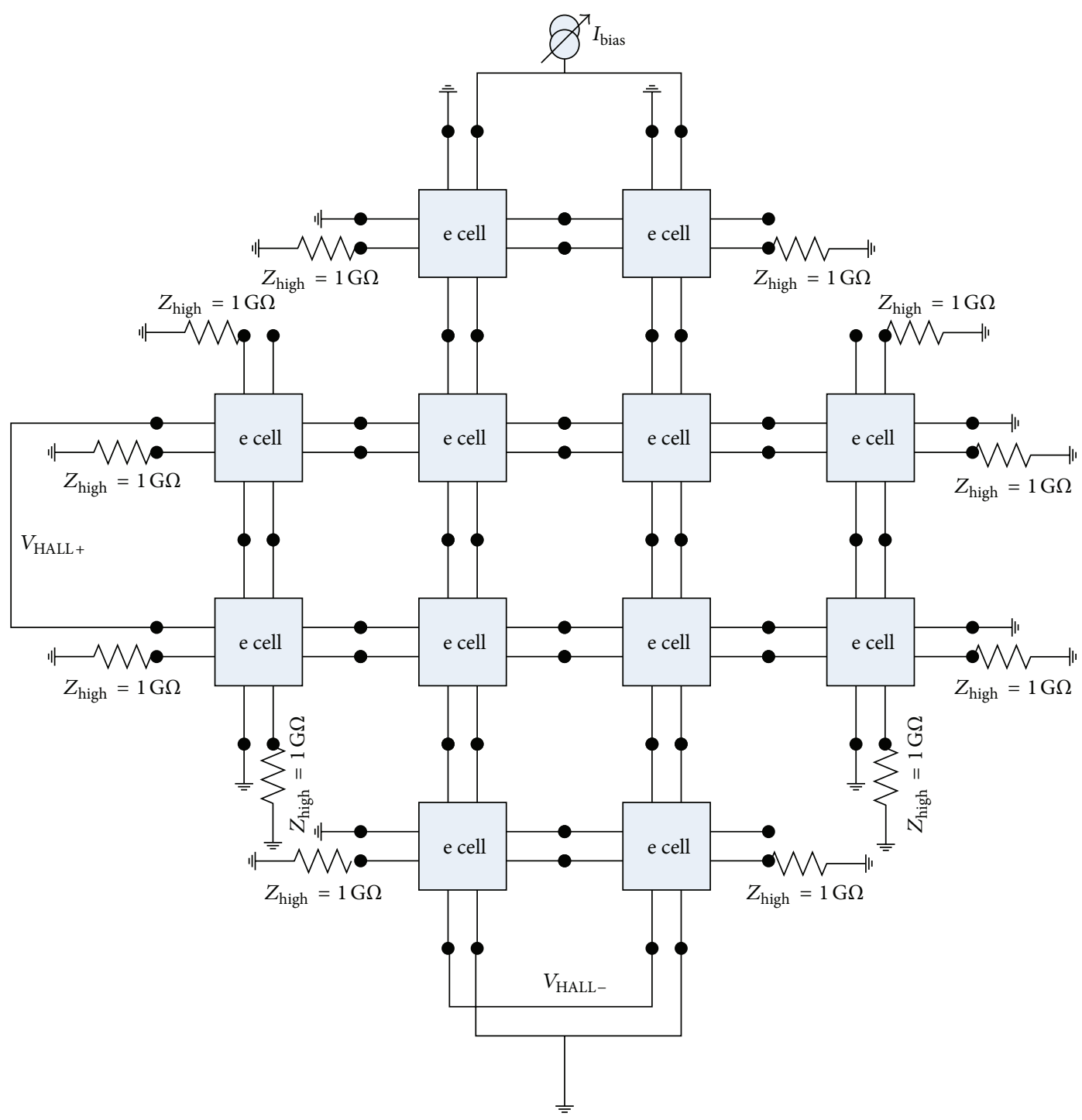

FIGURE 5: The XL cell representation by FEM model.

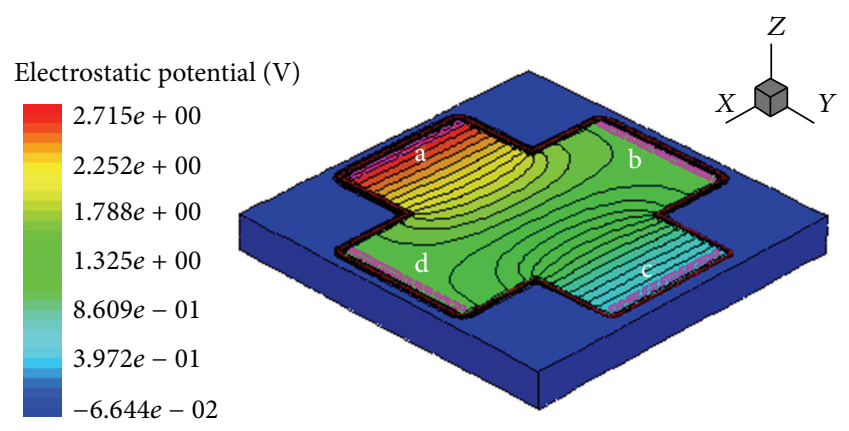

FIGURE 6: Three-dimensional representation of XL Hall cell with the electrostatic potential distribution.

and $T$ the absolute temperature in $\mathrm{K}$. In the case of silicon, $E_{g}=1.12 \mathrm{eV}$.
In Figure 7, the dependence of the carrier concentration $n$ with the reciprocal temperature $(1000 / T)$ and the doping concentration $N_{d}, n=n\left(N_{d}, 1000 / T\right)$ is represented in three dimensions.

The Hall scattering factor $r_{H}$ is defined as follows:

$$
r_{H}=\frac{\mu_{H}}{\mu}
$$

where $\mu_{H}$ is the Hall mobility and $\mu$ is the carrier mobility. Due to the fact that $r_{H}$ differs from the unity not only by scattering but also by anisotropy, this parameter is called simply Hall factor [10]. The Hall factor is a ratio of two mobilities which in turn obey a $T^{-\alpha}, \alpha>0$ temperature dependence in the considered temperature range where lattice scattering is prevalent.

Therefore, it is expected for $r_{H}$ to also have power dependence with the temperature. From the data provided in [11] for a impurity concentration close to the $N_{d}$ used in the 


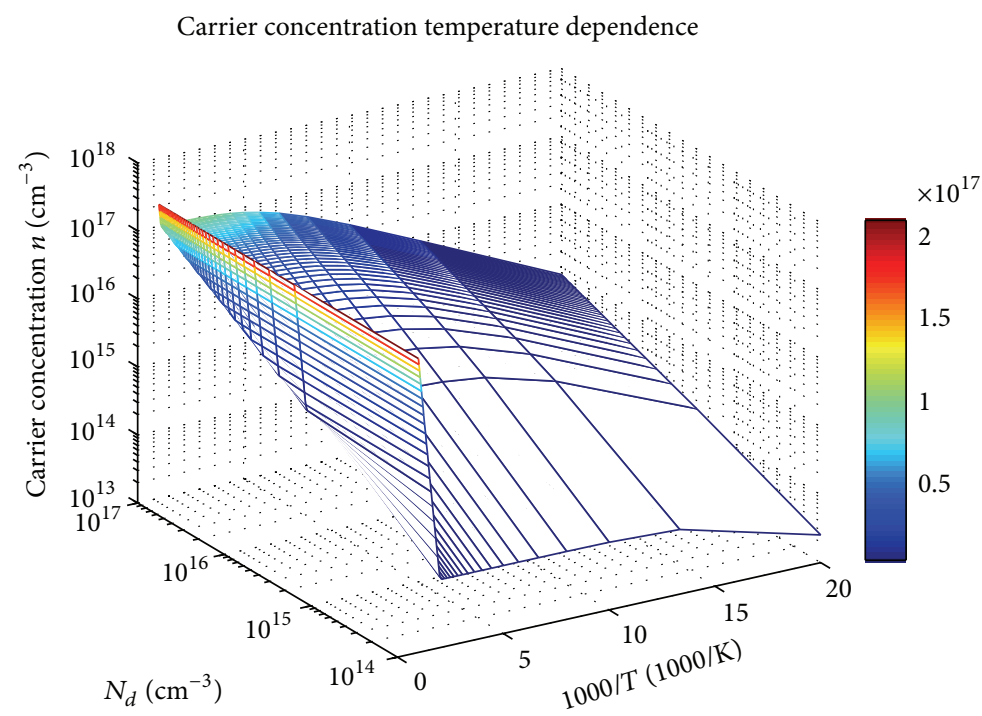

FIGURE 7: Graphical representation of carrier concentration $n$ versus reciprocal temperature and donor concentrations $N_{d}$.

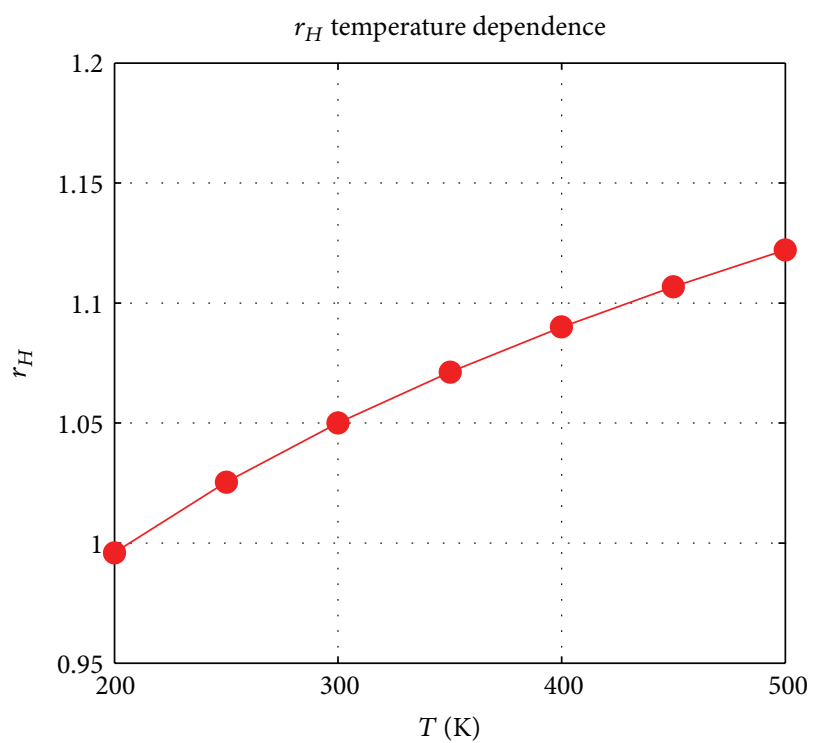

FIgURE 8: The Hall factor temperature dependence.

integration process, the dependence of $r_{H}$ with the temperature would obey, for this particular donor concentration, the following law:

$$
r_{H}=r_{H 0}\left(\frac{T}{T_{0}}\right)^{0.13}
$$

In (13), for impurity concentrations less than $10^{21} \mathrm{~m}^{-3}$, the Hall factor at $T_{0}=300 \mathrm{~K}$ is 1.15 . This value is almost equal to the Hall factor computed under spherical energy surface assumption and for a value of $3 \pi / 8=1.18$, only for the isotropic intravalley acoustic scattering [11].

However, in our case, for the absolute room temperature $T_{0}$, we use $r_{H 0}=1.05$, in accordance to the doping concentration $N_{d}=8.16 \cdot 10^{22} \mathrm{~m}^{-3}$ used in the Hall Effect cells integration process, to conform with the project parameters.

The temperature dependence of the Hall factor $r_{H}$ is represented in Figure 8, according to (13).

\section{Results and Discussion}

The finite element model developed in CADENCE containing the new proposed elementary cell was used to simulate different integrated Hall sensors. All additional blocks requiring modeling were coded in VERILOG-A. We can mention at this point that, even for a large number of elementary cells (for example a FEM model of the XL cell consisting of 64 elementary cells), the simulation time is reasonable, less than $1 \mathrm{~s}$, and the use of CPU resources is reasonable.

In this section, we validate the developed model by showing that there is good agreement between the simulated and measured data. Simulation results are given at this point for the XL, borderless, and optimum Hall cells, with the emphasis on the temperature behavior of the current-related sensitivity.

4.1. Hall Voltage and Sensitivity Simulation Results. The Hall voltage, absolute, and current-related sensitivity are some of the figures of merit predicted by simulation for the Hall devices. The temperature influence on the figures of merit governing the sensors performance was also extensively investigated.

Two integration CMOS processes were analyzed. The values of the parameters used to simulate the Hall cells within CMOS Process 1 are summed up in Table 2. Besides our integration process (Process 1), another CMOS process (Process 2) was simulated for the same cell. The data for Process 2 was taken from [6] and it is presented in Table 3.

Both voltage bias and current bias were used in the considered Hall cells simulation, but we are focusing at 


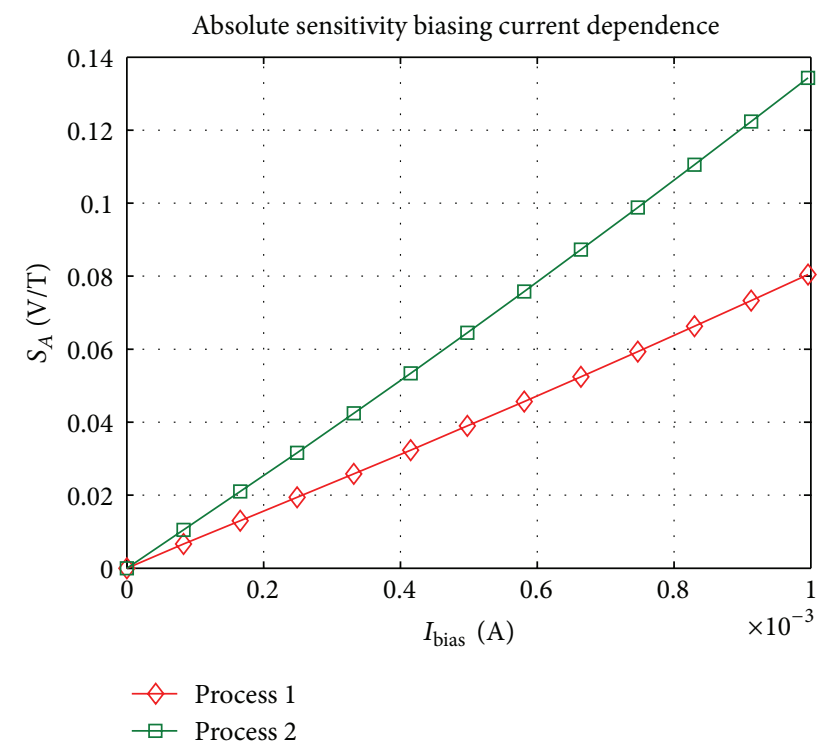

Figure 9: The simulated absolute sensitivity $S_{A}$ versus the biasing current for two integration CMOS processes.

TABLE 2: Process 1 parameters.

\begin{tabular}{lcc}
\hline Parameter & Symbol & Numerical value \\
\hline Length & $L$ & $43.2 \cdot 10^{-6} \mathrm{~m}$ \\
Width & $W$ & $22.6 \cdot 10^{-6} \mathrm{~m}$ \\
Thickness & $t$ & $10^{-6} \mathrm{~m}$ \\
Donor concentration & $N_{d}$ & $8.16 \cdot 10^{22} \mathrm{~m}^{-3}$ \\
Acceptor concentration & $N_{a}$ & $10^{21} \mathrm{~m}^{-3}$ \\
Conductivity & $\sigma$ & $933 \mathrm{Sm}^{-1}$ \\
Mobility & $\mu$ & $0.0715 \mathrm{~cm}^{-2} \mathrm{~V}^{-1} \mathrm{~s}^{-1}$ \\
Magnetic field & $B$ & $0.5 \mathrm{~T}$ \\
\hline
\end{tabular}

TABLE 3: Process 2 parameters.

\begin{tabular}{lcc}
\hline Parameter & Symbol & Numerical value \\
\hline Length & $L$ & $43.2 \cdot 10^{-6} \mathrm{~m}$ \\
Width & $W$ & $22.6 \cdot 10^{-6} \mathrm{~m}$ \\
Thickness & $t$ & $1.5 \cdot 10^{-6} \mathrm{~m}$ \\
Donor concentration & $N_{d}$ & $3.3 \cdot 10^{22} \mathrm{~m}^{-3}$ \\
Acceptor concentration & $N_{a}$ & $10^{21} \mathrm{~m}^{-3}$ \\
Conductivity & $\sigma$ & $382.8 \mathrm{Sm}^{-1}$ \\
Mobility & $\mu$ & $0.1 \mathrm{~cm}^{-2} \mathrm{~V}^{-1} \mathrm{~s}^{-1}$ \\
Magnetic field & $B$ & $0.5 \mathrm{~T}$ \\
\hline
\end{tabular}

this moment on current polarization. Figure 9 displays the simulated absolute sensitivity versus the biasing current obtained for the XL cell, in two integration processes.

Figure 10 presents the absolute sensitivity versus the biasing current for three of the simulated cells, XL, borderless, and optimum, respectively. For easier readability, the red line in Figure 10 corresponds to the same XL cell in Process 1 which was also simulated in Figure 9. We can see that the simulated results are in accordance with the measured data presented in Table 1.

As it was presented by authors in a recent paper, for the same integration process and current polarization, a maximization of the geometrical correction factor $G$ should be performed by the choice of the optimal shape. From formula in (2), we can observe that the Hall voltage is directly proportional to $G$, whose maximum value is 1 . For example, for the XL cell, $G=0.86$, a result which is in accordance with the theoretical value obtained through the formula in [12].

There is an increase of approximately $20 \%$ of the XL cell's absolute sensitivity with respect to the optimum cell. This is explained by the decrease of the optimum cell's absolute sensitivity due to the specific square structure with contacts located further away from the $\mathrm{p}-\mathrm{n}$ junction.

Figure 11 displays the simulated Hall voltage versus the magnetic field strength, for three integrated Hall cells. For $B=$ $0.5 \mathrm{~T}$, the simulated Hall voltage for XL cell is approximately $40 \mathrm{mV}$, while borderless and optimum cells yield $V_{\mathrm{HALL}}=$ $16.6 \mathrm{mV}$ and $V_{\mathrm{HALL}}=33.2 \mathrm{mV}$, respectively. The simulation results are in agreement with the measurement data.

4.2. Current-Related Temperature Behavior Simulation Results. In order to investigate the temperature drift of the current-related sensitivity, (10) and (11) for the carrier concentration, and (13) for Hall factor temperature dependence, respectively, were taken into account and fed into the equations governing the elementary cell behavior. From this perspective, we generated the current related sensitivity analytical function temperature dependence.

Simulations were performed in CADENCE to investigate the current-related sensitivity temperature dependence. The curve obtained in Figure 12 shows the current-related sensitivity parabolic temperature dependence for the XL cell. The considered temperature interval, from $-40^{\circ} \mathrm{C}$ to $120^{\circ} \mathrm{C}$, is the specific temperatures interval, range of interest for the industrial applications. For numerical reasons, in the absolute correspondent temperatures (measured in Kelvin), we use the covering interval between 240 and $400 \mathrm{~K}$.

The relative variation $\Delta S_{I} / S_{I}$ of the current-related sensitivity $S_{I}$ is introduced by the following equation, where $T_{0}$ is the absolute room temperature:

$$
\frac{\Delta S_{I}}{S_{I}}=\frac{S_{I}(T)-S_{I}\left(T_{0}\right)}{S_{I}\left(T_{0}\right)} .
$$

In Figure 13, the simulation has been performed in order to directly compare the results with the known experimental data.

The obtained graph in Figure 13(a) depicts the relative variation of the current-related sensitivity versus temperature for XL cell while Figure 13(b) shows the residuals after a fit of the simulated data with a second-order function.

Mathematically, the residual for a specific predictor value is the difference between the response value $y$ and the predicted response value $\hat{y}$. Assuming that the developed model fitted to the experimental data is correct, the residuals approximate the random errors. Practically, the residuals are the difference between the response data and the fit to the 


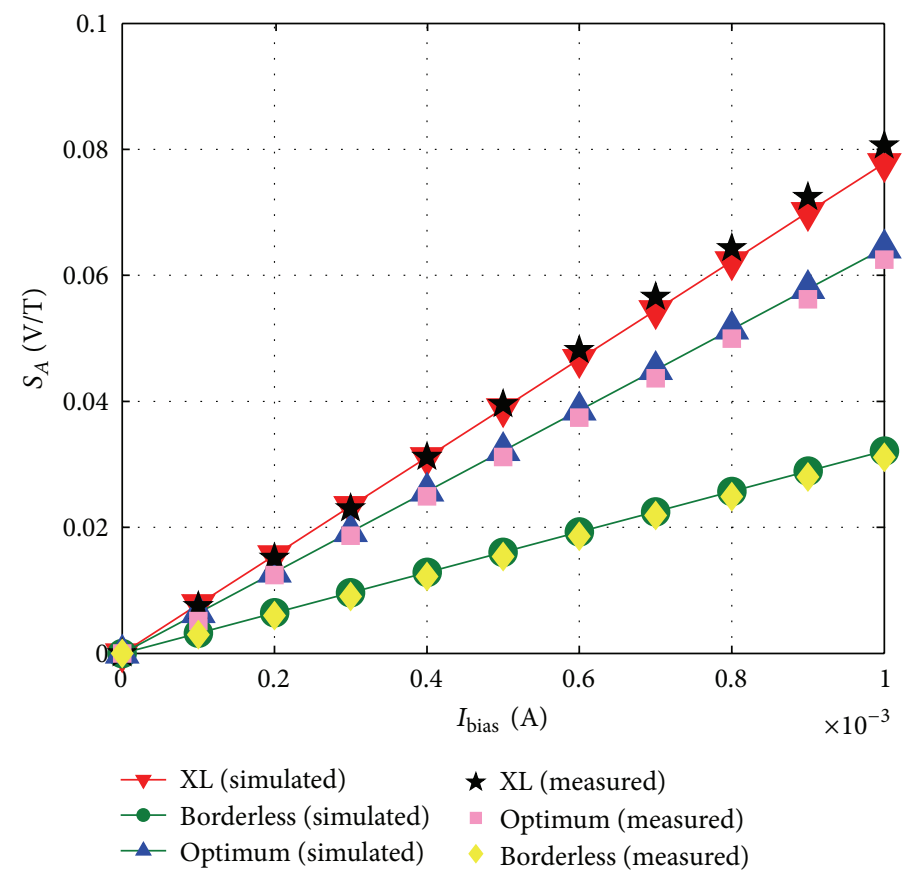

FIGURE 10: The simulated absolute sensitivity $S_{A}$ versus the biasing current for three integrated Hall cells.

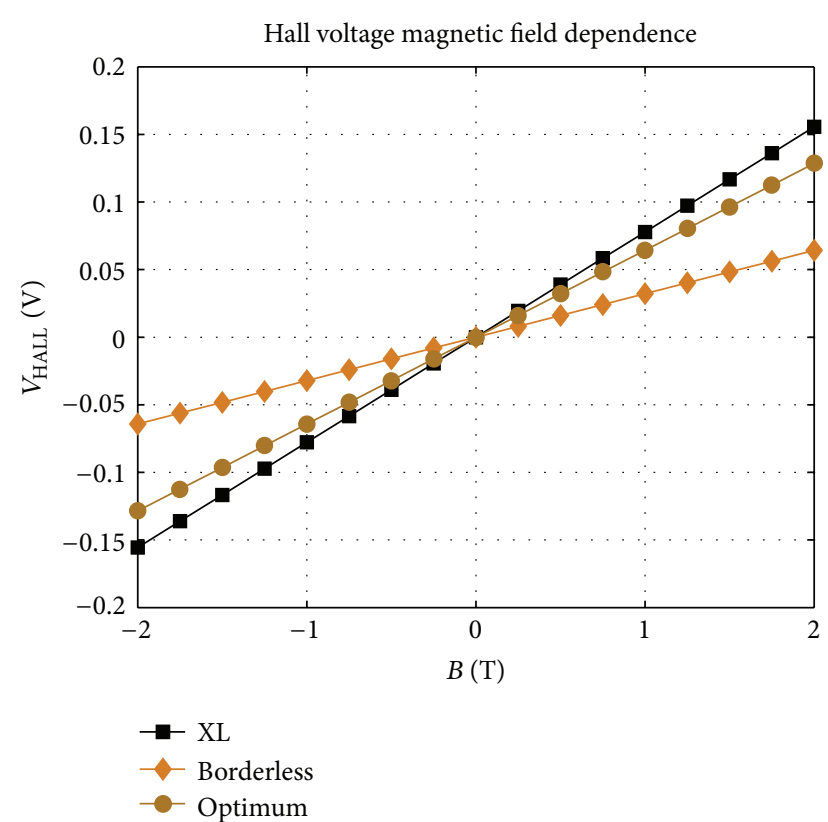

FIGURE 11: The simulated Hall voltage $V_{\text {HALL }}$ versus the magnetic field strength for three integrated Hall cells.

response data. In this case, the residuals are adimensional, as the initial fitted curve is a relative variation.

There is an excellent accordance of the simulations results obtained with the theoretical prediction and also with experimental results. The same parabolic allure, but for the temperature characteristic measurements of the "intrinsic" sensitivity (in fact the measured relative variation of the

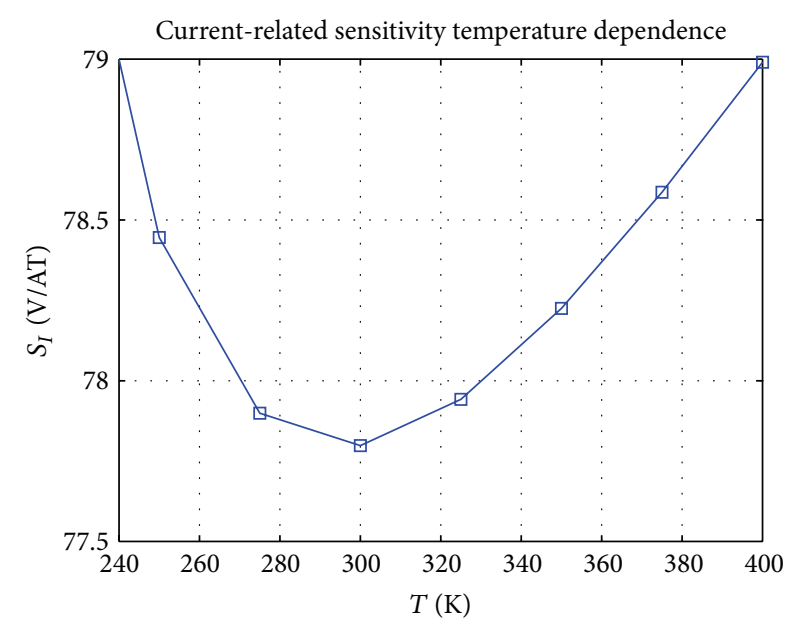

FIGURE 12: The simulated current-related sensitivity $S_{I}$ versus the temperature.

current-related sensitivity of the Hall plate), is announced by Manic [13] (except that the room temperature is $T_{0}=308 \mathrm{~K}$, compared with the generally accepted $T_{0}=300 \mathrm{~K}$ ).

We can mention that our simulations and both the measured and simulated relative variations of the "currentrelated sensitivity related to the value at room temperature" as a function of temperature, $S_{I}(T) / S_{I}\left(T_{0}\right)$, reported in [14], are also in good agreement.

The FEM model developed can be applied to a variety of Hall effect sensors shapes and different integration processes, by changing the specific parameters. However, the present model is destined to analyze horizontal Hall cells and it is not 


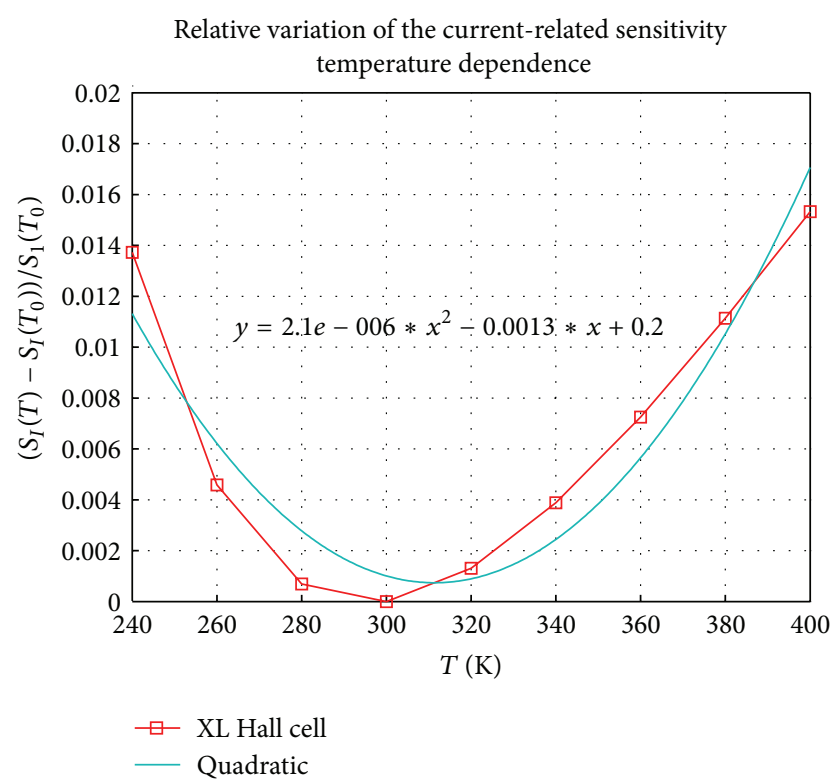

(a)

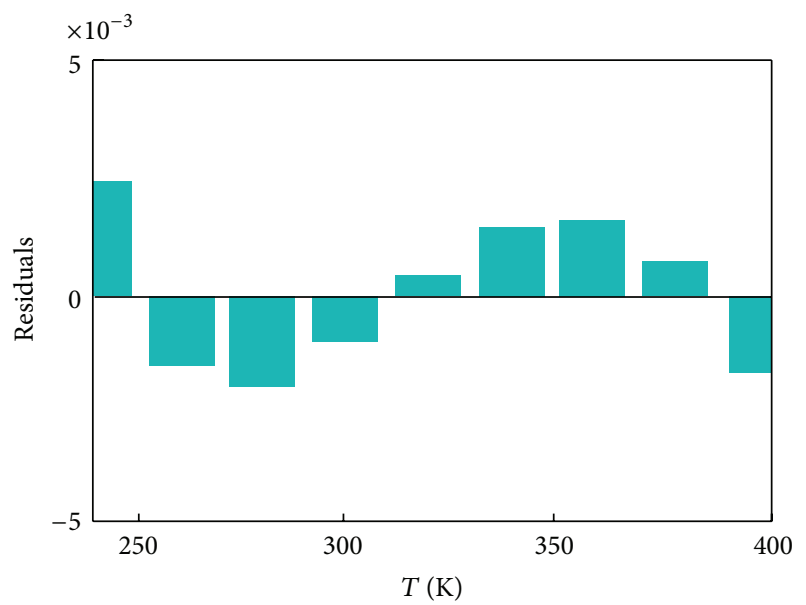

(b)

FIGURE 13: The simulated relative variation of the current-related sensitivity versus the temperature (a) and the residuals of the fitting curve (b).

valid at the moment for vertical sensors but can be changed, however, to serve this purpose, by changing the internal structure of the elementary cells, primary for the cells in the borders.

\section{Conclusion}

Different Hall effect sensors were integrated in a CMOS technology. The XL cell displayed the best results and proved to have the highest sensitivity and the minimum offset drift. To predict the performance of Hall effect sensors, a finite element lumped circuit model containing a new elementary cell with a slightly modified design was developed.

The proposed model implemented and tested contains both geometrical and physical parameters and is able to predict the Hall voltage, sensitivity, and their temperature drift. The temperature dependence of the Hall factor and the carrier concentration including freeze-out effect were carefully addressed by a detailed analytical analysis. In this way, the quadratic behavior of the current-related sensitivity with the temperature was also proven by simulation.

Simulations were performed for structures which reproduce the previously integrated Hall cells and the results obtained are in agreement with both the theory and the experimental results.

Due to the general character of applicability and versatility, even at this stage, our model can be used for other CMOS Hall effect devices integration processes, by adjusting the specific parameters such as doping concentration, conductivity, and thickness of the implantation profile.

In the future, after a specific calibration, the actual model will also be used for Hall effect sensors offset prediction and numerical evaluation.

\section{References}

[1] E. Ramsden, Hall-Effect Sensors-Theory and Applications, Elsevier, 2nd edition, 2006.

[2] M.-A. Paun, J.-M. Sallèse, and M. Kayal, "Geometry influence on the Hall effect devices performance," UPB Scientific Bulletin Series A, vol. 72, no. 4, pp. 257-271, 2010.

[3] M.-A. Paun, J.-M. Sallese, and M. Kayal, "Hall effect sensors performance investigation using three-dimensional simulations," in Proceedings of the 18th International Conference on Mixed Design of Integrated Circuits and Systems (MIXDES '11), pp. 450455, June 2011.

[4] M.-A. Paun, J.-M. Sallese, and M. Kayal, "Geometrical parameters influence on the Hall effect sensors offset and drift," in Proceedings of the 7th Conference on Ph.D. Research in Microelectronics and Electronics (PRIME '11), pp. 145-148, July 2011.

[5] R. S. Popovic, Hall Effect Devices, Institute of Physics Publishing, 2nd edition, 2004.

[6] P. D. Dimitropoulos, P. M. Drljaca, R. S. Popovic, and P. Chatzinikolaou, "Horizontal HALL devices: a lumped-circuit model for EDA simulators," Sensors and Actuators A, vol. 145146, no. 1-2, pp. 161-175, 2008.

[7] M. A. Paun, J. M. Sallese, and M. Kayal, "Comparative study on the performance of five different Hall effect devices," Sensors, vol. 13, no. 2, pp. 2093-2112, 2013.

[8] M. A. Paun, J. M. Sallese, and M. Kayal, "Hall effect sensors design, integration and behaviour analysis," Journal of Sensors and Actuator Networks, vol. 2, no. 1, pp. 85-97, 2013.

[9] M. A. Paun, J. M. Sallese, and M. Kayal, Hall cells offset analysis and modeling approaches [Ph.D. thesis], EPFL, Basel, Switzerland, 2013.

[10] M. Cornils, A. Rottmann, and O. Paul, "How to extract the sheet resistance and hall mobility from arbitrarily shaped planar fourterminal devices with extended contacts," IEEE Transactions on Electron Devices, vol. 57, no. 9, pp. 2087-2097, 2010. 
[11] E. Ohta and M. Sakata, "Temperature dependence of Hall factor in low-compensated n-Type Silicon," Japanese Journal of Applied Physics, vol. 17, no. 10, pp. 1795-1804, 1978.

[12] W. Versnel, "Analysis of the Greek cross, a Van der Pauw structure with finite contacts," Solid State Electronics, vol. 22, no. 11, pp. 911-914, 1979.

[13] D. Manic, J. Petr, and R. S. Popovic, "Temperature crosssensitivity of Hall plate in submicron CMOS technology," Sensors and Actuators A, vol. 85, no. 1, pp. 244-248, 2000.

[14] Y. Xu and H.-B. Pan, "An improved equivalent simulation model for CMOS integrated hall plates," Sensors, vol. 11, no. 6, pp. 6284-6296, 2011. 

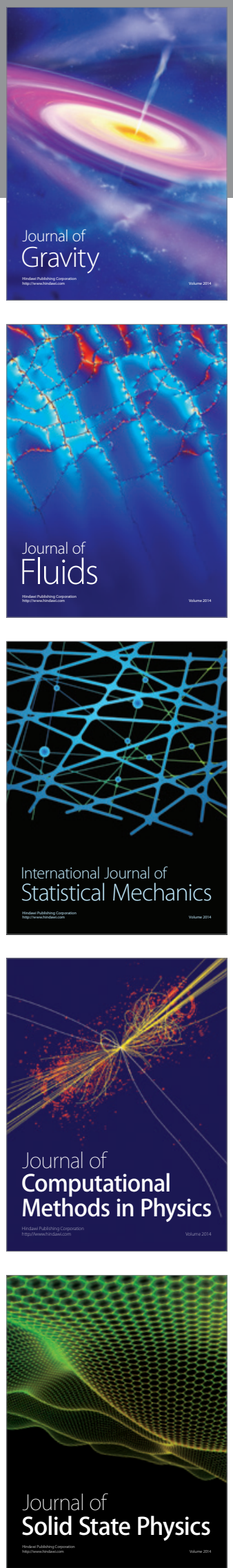

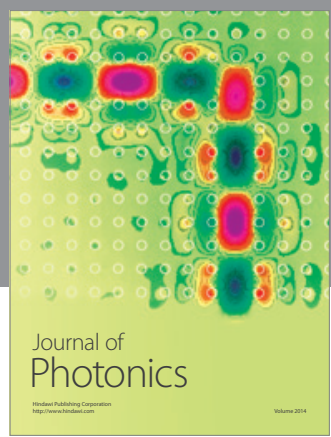

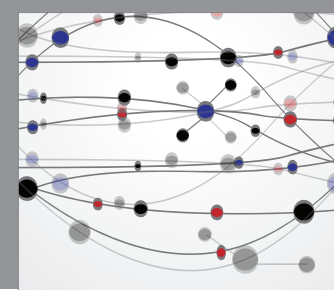

The Scientific World Journal

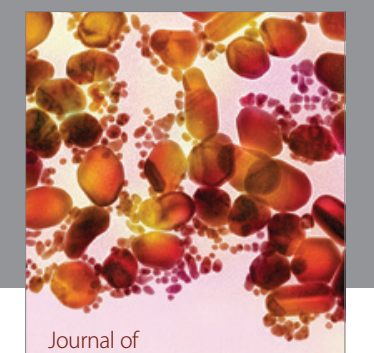

Soft Matter
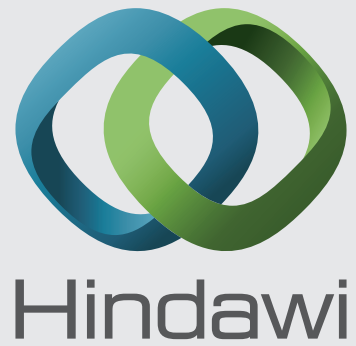

Submit your manuscripts at

http://www.hindawi.com
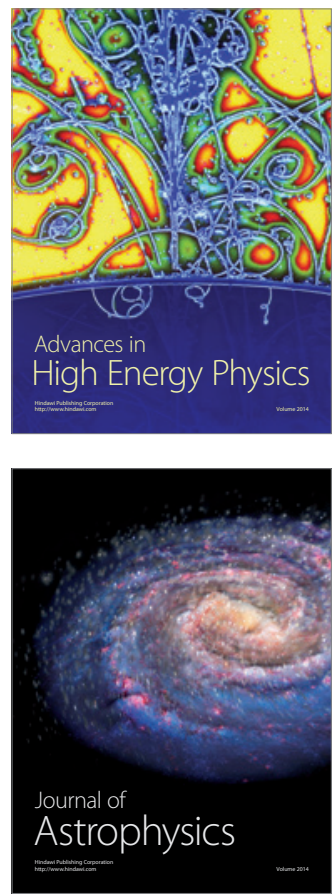
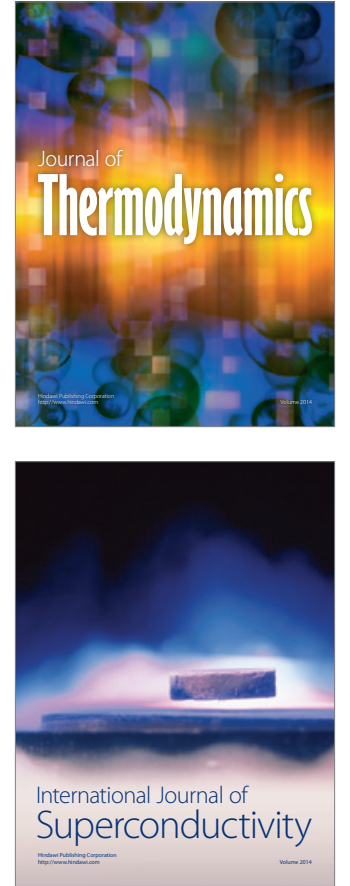
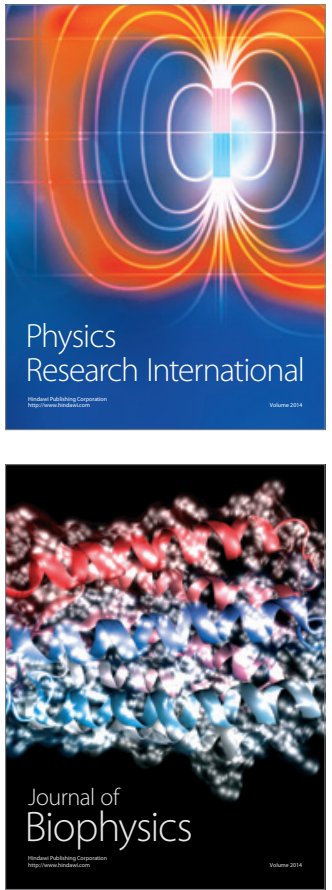
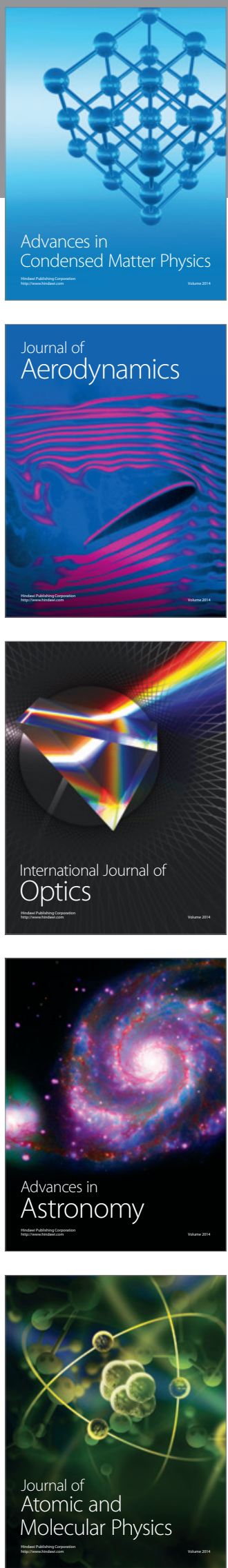